\title{
Método del caso como medio de evaluación dentro del modelo FLIP-Teaching. Una experiencia con una asignatura de corte jurídico para ingenieros.
}

\author{
Juan Vicente Oltra Gutiérrez ${ }^{\text {a }}$ \\ ${ }^{a}$ Escuela Técnica Superior de Ingeniería Informática. Universitat Politècnica de València. \\ jvoltra@omp.upv.es
}

\begin{abstract}
The following work presents an experience centered on the use of the case method as one of the evaluative methods to be used in the development of flip teaching, on a group of the subject Deontología y Profesionalismo, taught at the Escuela Técnica Superior de Informática (ETSINF) of the Universitat Politècnica de València.

The phases of the case method are described and the results obtained during the same are reported.
\end{abstract}

Keywords: Case Method, Flip Teaching, methodology, evaluation.

\begin{abstract}
Resumen
En el siguiente trabajo se presenta una experiencia centrada en el uso del método del caso como uno de los métodos evaluativos a utilizar en el desarrollo de la enseñanza inversa, sobre un grupo de la asignatura Deontología y Profesionalismo, impartida en la Escuela Técnica Superior de Informática (ETSINF) de la Universitat Politècnica de València.

Se describen las fases del método del caso y se da cuenta de los resultados obtenidos durante la misma.
\end{abstract}

Palabras clave: método del caso; clase inversa; metodología, evaluación.

\section{Introducción}

En el siguiente trabajo se presenta una experiencia centrada en el uso del método del caso como uno de los métodos evaluativos a utilizar en el desarrollo de la enseñanza inversa. El propósito último es avanzar hacia la alineación del eje enseñanza-aprendizaje-evaluación, cubriendo una meta a corto plazo: mejorar y documentar las mejoras de la experiencia que, sobre grupos de la asignatura Deontología y Profesionalismo, se viene realizando desde hace ya cuatro cursos académicos (OLTRA, 2015).

Usaremos indistintamente los términos enseñanza inversa y flip teaching, por el extendido uso de ambos y, porque un reciente estudio bibliométrico (inédito, pendiente de publicación en el congreso INNODOCT 18) nos indica que ambas expresiones son empleadas de forma similar en la producción científica en español (40\%-60\% aproximadamente). 


\section{Definición de los términos de partida. Flip Teaching.}

El modelo Flip Teaching, según (SEIN-ECHALUCHE, 2017) comenzó con el intercambio del lugar donde habitualmente se realizan dos de las principales actividades educativas: "la lección y los deberes” cifrando su punto de partida como método en el año 2000. Este año como punto de partida toma como origen la iniciativa de un grupo de profesores de economía en la Universidad de Miami (Ohio) (VIDAL MELÓ, 2016), aunque de su forma conocida hoy no aparece registro hasta 2007, cuando Jonathan Bergmann y Aaron Sams, profesores de química en Woodland Park High School, Colorado, empezaron a grabar en soporte video los contenidos de sus asignaturas y a subirlos a la red y relacionarlos con una serie de trabajos a realizar en casa sobre los mismos liberando el tiempo de aula para resolver dudas y realizar actividades aumentando la interacción profesor-alumno. (VIDAL MELÓ, 2017).

Otros autores que analizan el fenómeno antes de ese año (ANDREU, 2004), sin embargo se remontan a 1870, entendiendo como un claro precedente la experiencia de Chistopher Laudell en los estudios de derecho de la Universidad de Harvard. La definición del método está sujeta a muchas variantes en los detalles, aunque en lo elemental podríamos decir, siguiendo a (SEIN-ECHALUCE LACLETA, 2015) que, frente al modelo de formación habitual basado en impartir lecciones magistrales en el aula, con un alumnado pasivo, que ocasionalmente puede ser empujado a realizar actividades fuera del horario presencial, lo que se trata es de invertir el proceso y que el muy escaso tiempo de aula se emplee para trabajar sobre contenidos teóricos, que el alumno ha debido adquirir antes de asistir. Así, para provocar que fuera de clase ocurra la transferencia de conocimientos, el alumno contará con una serie de recursos dejando el tiempo de aula para la asimilación de los mismos. (CANALES VARGAS, 2014)

\section{Definición de los términos de partida. Método del caso.}

Mientras el flip teaching es algo relativamente novedoso, el método del caso es un viejo conocido para los docentes. Con él, buscamos provocar el análisis y la resolución de una situación que carece de solución única, compleja y a menudo con variantes que presentan dilemas éticos, a través de la puesta en común con el discente quien, mediante la reflexión y el diálogo, toma decisiones a la luz de la teoría que el docente le presentó, y así trata de resolver los problemas planteados, de manera colaborativa.

Hay múltiples experiencias ((CASTELLÓ-SIRVENT, 2017), (CANALES VARGAS, 2014) entre otros) pero básicamente en todas el guión es el mismo: los alumnos confrontan informaciones reales, incluso en ocasiones en apariencia contradictorias, en las que se apoyarán para resolver el problema o problemas planteados, con un apoyo no solo basado en los materiales preparados para la actividad en particular, sino con el obligado contraste con el contenido teórico de la asignatura.

\section{Objetivos.}

Si tuviéramos que definir un único objetivo, sin duda señalaríamos el intentar la consecución de ese término que entre utópico y acariciado aparece recurrentemente en la 
mente de todo docente: la evaluación continua, equilibrando enseñanza, evaluación y aprendizaje.

Aplicando el método del caso tratamos de efectuar un alto nivel de transferencia de conocimientos. Para ello nos apoyamos en casos reales, que el alumno puede conocer a través del material que el profesor le suministra, pero también a través de sus propios rastreos en buscadores generalistas o especializados (Aranzadi, AENOR...)

Al tratarse de acontecimientos donde profesionales informáticos se han visto implicados, en algunos casos incluso con consecuencias penales, se focaliza la atención del alumno permitiendo una participación activa del mismo. Más allá de acercar la teoría a la realidad, el propósito es acercar la realidad a la teoría. Realidad que el alumno puede contrastar por sí mismo, y en algún caso incluso forma parte de su memoria reciente (pues se trata de emplear casos no solo relevantes sino, en la medida de lo posible muy mediáticos y próximos en el tiempo).

Consideramos, con (ANDREU, 2004), que el aprendizaje precisa de una participación activa del estudiante en lugar de esperar a que el profesor desarrolle de forma teórica el contenido. El objetivo último es fomentar la curiosidad y el desarrollo de destrezas que permiten el autoaprendizaje a lo largo de toda la vida.

Una relación más amplia, y por tanto ambiciosa, de los objetivos, incluiría

- El mejor aprovechamiento del tiempo de aula en busca de unas clases más productivas

- Apoyándonos en el interés que el alumno (estudiante de Grado en Informática) tiene por las tecnologías de la información y la comunicación, explotar de forma extensa las oportunidades que las herramientas que nos proporciona la universidad, nos ofrece.

- Propiciar búsquedas de información que tradicionalmente no pueden hacerse en el aula por falta de tiempo o de medios, de forma que desde su domicilio, en un lugar de trabajo compartido con otros compañeros, alcancen mayor profundidad de la que el docente podría exponer en una clase presencial. Esto, abunda en la idea de lograr mayor creatividad en profesores y alumnos.

- En suma, evadir las debilidades típicas del modelo puramente presencial, la monotonía y el uso de un tiempo que se nos escapa entre las manos, dando el volante al propio alumno, que pasa de ser pasajero a conductor en el itinerario de su aprendizaje.

\section{Desarrollo de la experiencia.}

La experiencia se desarrolla en el único grupo de la asignatura Deontología y Profesionalismo perteneciente al doble grado en Informática y Administración de Empresas, durante el primer semestre, código 11550, en adelante DyP. Esta asignatura tiene otros siete grupos en el segundo semestre, dentro del grado de Informática. El número y características principales de los alumnos pueden verse en la tabla 1. 
Con respecto al perfil de los alumnos participantes, hay que añadir a los datos objetivos recogidos en la tabla que tienen un perfil que se diferencia del resto de alumnos que cursan DyP, en el segundo semestre, matriculados en el grado de informática. La diferencia más cuantificable la tenemos en su nota de acceso a la universidad (3,3 puntos mayor para los primeros), además de que cursan la materia en el primer semestre de su tercer curso, en lugar de en el segundo semestre de segundo curso. Sus notas son ostensiblemente más altas que la media (se triplica el porcentaje de sobresalientes, se duplica el de notables y se reduce a cero el número de suspensos y no presentados).

Tabla 1. Características de los participantes.

\begin{tabular}{|lll|}
\hline Asignatura / características de los alumnos & DyP & \\
\hline Número de alumnos reales & 29 & \\
\hline Alumnos que simultanean trabajo con estudios & 1 & $3,5 \%$ \\
\hline Rango de edad (años) & $20-21$ & \\
\hline $\begin{array}{l}\text { Alumnos con conocimientos previos de la materia } \\
\text { Alumnos emancipados (viven solos o con su familia, asumiendo una } \\
\text { parte significativa de los gastos de la vivienda, sin financiación } \\
\text { familiar) }\end{array}$ & 0 & $0 \%$ \\
\hline
\end{tabular}

Fuente: Elaboración propia

La asignatura DyP trata de aproximar los contenidos relativos a la profesión informática (marcos profesionales empleados en la Unión Europea), legislación informática más relevante y el marco deontológico de la profesión informática a los alumnos, contando para ellos con 4,5 créditos ECTS. Los datos más relevantes para caracterizar a la asignatura y sus métodos de evaluación pueden verse en la tabla $n^{\circ} 2$.

Tabla 2. Características de las asignaturas.

\begin{tabular}{ll}
\hline Asignatura / características & DyP \\
\hline Créditos totales de teoría & 3 \\
\hline Créditos totales de práctica & 1,5 \\
\hline Inicio de clases & $5 / s e p t$ \\
\hline Fin de clases & $22 /$ dic \\
\hline Duración de una clase de teoría (en horas) & 2 \\
\hline $\mathbf{N}^{0}$ de clases de teoría a la semana & 1 \\
\hline $\mathbf{N}^{\mathbf{0}}$ de clases de teoría en el semestre & 13 \\
\hline
\end{tabular}




\begin{tabular}{|ll|}
\hline Asignatura / características & DyP \\
\hline $\mathbf{N}^{\circ}$ de tareas que se evalúan & 3 \\
\hline Peso de las tareas en la nota de la asignatura & $30 \%$ \\
\hline $\mathbf{N}^{0}$ de casos de aula con test que se evalúan & 5 \\
\hline Peso de los casos evaluados en la nota de la asignatura & $35 \%$ \\
\hline $\mathbf{N}^{0}$ de casos de aula totales que se realizan & 7 \\
\hline $\mathbf{N}^{0}$ de trabajos de prácticas que se evalúan & 4 \\
\hline Peso de los trabajos de prácticas en la nota de la asignatura & $35 \%$ \\
\hline
\end{tabular}

Anticipábamos que tratamos con la presente experiencia de eliminar la inactividad del alumnado en el aula, idea fundamental en el flip teaching, empleando para ello, entre otros elementos, el método del caso.

En el mismo, presentamos de una serie de elementos factibles y/o verídicos buscando provocar la reflexión del alumnado. Para ello, primero se presenta un problema que luego es analizado y, en algunas situaciones, resuelto.

Basados en hechos reales, preferentemente noticias de actualidad y de impacto relevante en la profesión, además de transmitir hechos o datos importantes nos permite potenciar el pensamiento crítico en el alumnado. Con (MENENDEZ, 2003) incidimos en lo prioritario de que el caso se apoye en la vida profesional actual intentando recrear los condicionantes reales en la práctica profesional

Las fases en que se descompone el desarrollo del mismo son:

1. Compartir (generalmente mediante "recursos" en la plataforma educativa) la narración del caso y elementos de apoyo. Se les indica que contenidos teóricos están relacionados y se les sugiere también búsquedas de material de apoyo. En este momento, se hacen públicos los objetivos de aprendizaje del caso y, con ellos en cuenta, se da a conocer el mecanismo de evaluación y que evidencias permitirán cuantificar ésta. A la selección del caso más adecuado hay que sumar la programación previa, esto es: estudiar en qué momento de la asignatura se realiza (no solo para que los contenidos teóricos hayan podido ser vistos, sino para evitar colapsos de agenda en los alumnos, intentando no coincidir con periodos intensos de entregas por parte de éstos).

2. Antes del trabajo en aula, se les indica a los alumnos la necesidad de una lectura y análisis individualizado de los documentos compartidos.

3. Debate y discusión en aula. El grupo se divide en subgrupos de trabajo (de un tamaño de 4 a 6 miembros). Dado que emplearemos en algunos casos test rápidos de respuesta para 
evaluar el caso, provocamos que se planteen preguntas, de forma que esta aportación de los alumnos y sus informes permitan construir el propio elemento de evaluación. Esto es: se les evaluará en buena medida con sus propias preguntas. Durante este debate se aplican técnicas de gamificación que permiten al profesor animar el mismo y motivar a los presentes. El debate se utiliza para introducir dilemas éticos. Durante el mismo el docente actúa no solo como conductor, sino que va interpretando los roles de facilitador de información, mediador en caso de conflictos, orientador si surgen dudas sobre el aparato teórico, etc., además de controlar la participación en forma y fondo de los alumnos en el mismo.

4. Evaluación. Una vez acabado el trabajo en aula, para determinados casos se abre un pequeño examen en la plataforma educativa. En el resto de ellos, la única evaluación es la procedente de la gamificación (sobre la gamificación cabe indicar en este momento que la descripción de este proceso y los datos asociados de forma completa se han presentado. en una comunicación a INRED 20178, estando la misma pendiente de aprobación)

Recordemos que no buscamos una solución concreta, pues muchas veces esta no es única y de las posibles, los matices éticos de cada una pueden hacerlas más o menos adaptables por los distintos grupos.

Un ejemplo de una instanciación concreta lo podemos ver en la figura siguiente.

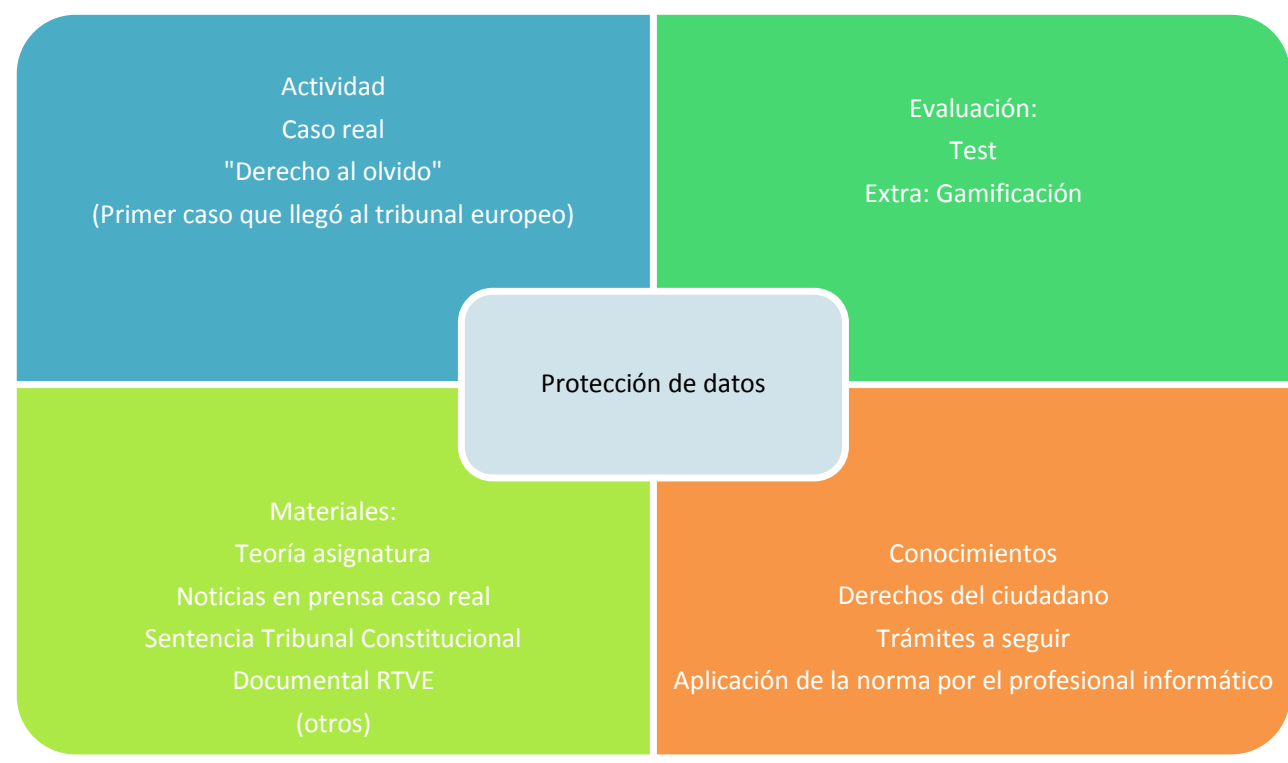

Fig. 1 Caso "Derecho al olvido", resumen gráfico. 


\subsection{Ficha del caso.}

Por cada uno de los casos, el profesor genera una ficha, tanto para apoyo propio como para posibilitar la reutilización del caso por otros docentes, basado en el trabajo de (REYNOLDS, 1992).

En ella, se identifica el caso por su título y temas de teoría relacionados, indicando los objetivos didácticos perseguidos y un cálculo del tiempo de trabajo que tanto dentro como fuera del aula va a conllevar a los alumnos.

Indicamos los aspectos esenciales, como la bibliografía recomendada y el trabajo recomendado con plazos dados a los alumnos, tanto dentro como fuera del aula.

A modo de guía para el docente se indican unas guías de actuación para el debate (elementos que aparecen con frecuencia, por ejemplo), así como distintas soluciones y sugerencias aportadas por los alumnos en situaciones precedentes, si las hay (recuérdese que se trata de emplear casos de rabiosa actualidad)

Puede verse en la fig.2 un ejemplo de la ficha de apoyo para el docente

Ficha del caso "Muerto pixelado"

Temas de teoría relacionados: Tema 5 DyP, "Protección de Datos"

Objetivos didácticos perseguidos: Diferenciación entre protección de datos y derecho al honor. Definición de responsabilidad profesional del encargado del tratamiento de datos.

Cálculo del tiempo de trabajo: 30 minutos en aula. 30 minutos en casa.

Bibliografía recomendada: apuntes de la asignatura. Reglamento Europeo de Protección de Datos.

Plazos: La tarea se entrega a la semana de su apertura.

Guía de actuación para el profesor: refrescar los conceptos de dato personaly derivados. Cambios legales en la protección de datos de fallecidos. Es importante observar que con el marco legal anterior la situación variaba diametralmente a este respecto.

Fig. 2 Ficha de un caso. Elaboración propia.

\section{Resultados.}

Al buscar elementos cuantificables nos encontramos con dos posibilidades: medir la actividad en sí (mediante el acceso a los recursos y la participación en aula) y los resultados de la evaluación de la misma: las notas.

En la tabla siguiente podemos ver la relación completa de casos. Vemos la participación premiada en el proceso de gamificación, la nota máxima que se podía obtener en el examen planteado y dos valores: el de los alumnos suspendidos y el de los alumnos con una nota que supera el $80 \%$ del valor que podría obtenerse. El acceso medio a los recursos por 
alumno ha sido de unas 109 interacciones, lo que se corresponde muy bien con el acceso a los recursos disponibles, de forma unitaria.

Tabla 3. Calificaciones obtenidas por los alumnos en el método del caso.

\begin{tabular}{rlrrrr}
\hline Caso (no) & $\begin{array}{l}\text { Peso } \\
\text { (puntos) }\end{array}$ & $\begin{array}{l}\text { Gamificación } \\
\text { (participación) }\end{array}$ & Nota media & Suspendidos & $\begin{array}{c}\text { Nota } \\
>80 \%\end{array}$ \\
\hline $\mathbf{1}$ & 0 & $24 \%$ & & & \\
$\mathbf{2}$ & 0,5 & $21 \%$ & 0,38 & $0 \%$ & $66 \%$ \\
$\mathbf{3}$ & 1 & $45 \%$ & 0,65 & $17 \%$ & $31 \%$ \\
$\mathbf{4}$ & 0 & $24 \%$ & & & \\
$\mathbf{5}$ & 0,5 & $24 \%$ & 0,33 & $21 \%$ & $41 \%$ \\
$\mathbf{6}$ & 1 & $45 \%$ & 0,68 & $3 \%$ & $41 \%$ \\
$\mathbf{7}$ & 0,5 & $24 \%$ & 0,22 & $41 \%$ & $17 \%$ \\
\hline
\end{tabular}

Esta tabla denota un gran seguimiento y un éxito en líneas generales en la evaluación, con algún "pinchazo" como el producido durante el último caso (realizado ya muy próximos los exámenes y con los alumnos pensando más en estos que en otras actividades). Podemos comprobar esto con la tabla siguiente, que nos muestra las finales de la asignatura (donde, recordemos, los casos implican un 35\% de la nota total)

Tabla 4. Notas finales.

\begin{tabular}{lcc}
\hline & DyP & DyP (\%) \\
\hline MH & 1 & $3,44 \%$ \\
NB & 9 & $31,03 \%$ \\
AP & 17 & $58,62 \%$ \\
SP & 2 & $6,89 \%$ \\
No presentados & 0 & $0 \%$ \\
PRES & 0 & $0 \%$ \\
NO PRES & 29 & \\
NOTA MEDIA & 0 & $(10-5)$ \\
\hline
\end{tabular}

\section{Conclusiones}

Aplicando esta técnica grupal fomentamos la participación del alumno, desarrolla el espíritu crítico y nos ayuda a prepararle para la toma de decisiones el contraste de 
opiniones con los demás componentes del grupo. Además nos permite tomar el pulso a la clase, y comprobar de primera mano que conceptos necesitan un refuerzo y cuales se encuentran asumidos. Por otra parte, al provocar la confrontación de puntos de vista opuestos abre unas posibilidades estupendas para la evaluación de competencias como la ética o el pensamiento crítico.

Al permitir contrastar la realidad con la teoría, facilitamos que el alumnado centre su atención, pues los contenidos de la asignatura dejan de ser algo difuso para convertirse en algo que está pasando, que es noticia de telediario.

Los resultados obtenidos en las calificaciones y los datos de participación nos muestran una mayor implicación y éxito en las pruebas que alumnos de grupos con metodologías docentes convencionales. Las notas globales de la asignatura, significativamente más altas, parecen apuntar (algo que habría que precisar en posteriores estudios considerando grupos con estructura análoga de alumnos por origen y temporización docente) a un efecto claramente positivo de las estrategias empleadas.

También para posteriores experiencias se plantea la preparación de un pequeño cuestionario de satisfacción.

Por último, el dato elevado de resultados obtenidos en la gamificación, recordemos que como actividad voluntaria, subraya siquiera sea desde criterios subjetivos la idea de que la actividad despierta el interés en el alumnado.

\section{Referencias}

AKEMI IKEDA, A.; MODESTO VELUDO-DE-OLIVEIRA, T. CORTEZ CAMPOMAR, M. (2005). “O caso como estratégia de ensino na áreade Administração”. En Revista de Administraçao -RAUSP, Universidade de São Paulo. São Paulo, Brasilvol. 41, núm. 2, abril-junio, 2006, pp. 147-157. http://www.redalyc.org/articulo.oa?id=223417482003 [Consulta: 18 de marzo de 2018]

ANDREU, Mª́ Á; GONZÁLEZ, J.A.; LABRADOR, Mª J.; QUINTANILLA, I.; RUIZ, T. (2004). “Método del caso. Ficha descriptiva y de necesidades" UPV. http://www.upv.es/nume/descargas/fichamdc.pdf [Consulta: 18 de marzo de 2018]

CANALES VARGAS, G. (2014). "Medio ambiente, ciencia y valores". En XI Jornadas Internacionales de Innovación Universitaria, Villaviciosa de Odón, 7-8 de Julio, 2014.<http://hdl.handle.net/11268/3767>[Consulta: 18 de marzo de 2018]

CASTELLÓ-SIRVENT, F.; CRISTINA SANTOS ROJO, C. (2017);” Aplicación del método del caso en el EEES: el ere de Coca-Cola” En Revista Jurídica de Investigación e Innovación Educativa. № 16, junio 2017. Universidad de Málaga. Málaga. 35-55 $<$ https://ideas.repec.org/a/erv/rejiee/y2017i1605.html>[Consulta: 18 de marzo de 2018]

MENENDEZ, J.M., GUIRAO, B., RIVAS, A. Y NIÑO, A. (2003). “La enseñanza de la Planificación del Transporte mediante el análisis de casos”. Actas del I Encuentro Internacional de Enseñanza de la Ingeniería Civil, 18-20 de septiembre, Ciudad Real. (CITADO)

OLTRA GUTIÉRREZ, J.V. (2015). "Diseño de una experiencia de Flip-Teaching para la asignatura Deontología y Profesionalismo a impartir en la Escuela Técnica Superior de Ingeniería Informática de la UPV" En INRED 2015. Valencia, UPV.

$<$ http://ocs.editorial.upv.es/index.php/INRED/INRED2015/paper/viewFile/1646/484>[Consulta: 18 de marzo de 2018] 
REYNOLDS, J.I. (1992). "El método del caso y la formación en gestión. Guía práctica". Valencia: Instituto de la Pequeña y Mediana Industria Valenciana.

SEIN-ECHALUCE LACLETA, M. L.; FIDALGO BLANCO, A.; GARCÍA PEÑALVO, F. (2015) “Metodología de enseñanza inversa apoyada en b-learning y gestión del conocimiento" En III Congreso Internacional sobre Aprendizaje, Innovación y Competitividad (CINAIC 2015). Zaragoza. Universidad de Zaragoza. 464-468

SEIN-ECHALUCE. M.L. FIDALGO-BLANCO, A. GARCÍA-PEÑALVO, F.J. (2017)“Trabajo en equipo y FlipTeaching para mejorar el aprendizaje activo del alumnado” En IV Congreso Internacional sobre Aprendizaje, Innovación y Competitividad (CINAIC 2017). Zaragoza. Universidad de Zaragoza. 610615.

VIDAL MELÓ, A.; ESTRUCH FUSTER, V.D.; BOIGUES PLANES, F. J. (2017). “Flipped Teaching aplicado al estudio de los métodos elementales de integración aproximada. Una experiencia educativa” En ROIG-VILA, R. (coord.). Redes colaborativas en torno a la docencia universitaria. Alicante: Universidad de Alicante, Instituto de Ciencias de la Educación (ICE), 2017. ISBN 978-84-617-8973-3, 571 p. $<$ http://hdl.handle.net/10045/70977>[Consulta: 18 de marzo de 2018]

VIDAL MELÓ, A.; ESTRUCH FUSTER, V.D.; BOIGUES PLANES, F.J. DEL REY TORMOS, R.; ALBA, J.; ROIG SALA, B.; TORREGROSA, C. (2016). "Flipped Teaching: una metodología en construcción..." En INRED 2016. Valencia, UPV.

$<$ https://www.researchgate.net/publication/305026754_Flipped_Teaching_una_metodologia_en_constr uccion>[Consulta: 18 de marzo de 2018]

(c) EY-Nc-ND 2018, Universitat Politècnica de València 